\title{
How to reduce a genome? ALife as a tool to teach the scientific method to school pupils
}

\author{
Quentin Carde $^{1}$, Marco Foley ${ }^{1}$, Carole Knibbe ${ }^{1,2}$, David P. Parsons ${ }^{1}$, \\ Jonathan Rouzaud-Cornabas ${ }^{1,3}$ and Guillaume Beslon ${ }^{1,3}$ \\ ${ }^{1}$ Inria Beagle Team, F-69603, France \\ ${ }^{2}$ Univ. Lyon, CarMeN Lab., INSERM, INRA, INSA-Lyon, Univ. Claude Bernard Lyon 1, F-69621, Villeurbanne, France \\ ${ }^{3}$ Univ. Lyon, LIRIS Lab., INSA-Lyon, CNRS, UMR5205, F-69621, France \\ guillaume.beslon@inria.fr
}

\begin{abstract}
When Artificial Life approaches are used with school pupils, it is generally to help them learn about the dynamics of living systems and/or their evolution. Here, we propose to use it to teach the scientific and experimental method, rather than biology. We experimented this alternative pedagogical usage during the 5 days internship of a young schoolboy - Quentin - with astonishing results. Indeed, not only Quentin easily grasped the principles of science and experiments but meanwhile he also collected very interesting results that shed a new light on the evolution of genome size and, more precisely, on genome streamlining. This article summarizes this success story and analyzes its results on both educational and scientific perspectives.
\end{abstract}

\section{Introduction}

In France, the school program for teenagers aged 14 or 15 includes a 5-day internship in a professional environment. The goals of this internship are (i.) to discover the economic and professional world, (ii.) to have the pupils face the concrete realities of employment and (iii.) to help them build their professional project. Pupils are often welcomed in a relative's company. Children of researchers are no exception and they are often welcomed in a laboratory of their parents' university. During these internships in laboratories, it is generally considered that the actual scientific work is out of reach for the pupils, either because they don't have the necessary background or because the internship is not long enough. Hence the pupils generally visit various teams, discuss with the researchers and with the technical and administrative staff without discovering the reality of research.

In November 2018, one of us was contacted by the father of a young pupil, Quentin, who wished to discover the job of researcher while his family had no background and no contact in this domain. We agreed to welcome Quentin in the team and proposed that it would be a real professional internship, i.e., that Quentin would conduct his own, real, research project during his stay. We proposed to Quentin to discover the reality of the research work, from the statement of a scientific questioning to the analysis of experimental results. On our side, the idea was that artificial life could make it possible to carry out a research project, even in a the very limited time-frame of five days, with little prior knowledge and no previous experimental practice.

Quentin finally completed his internship in the Beagle team from Monday, January $28^{\text {th }}$ to Friday, February $1^{\text {st }}, 2019$ under the direct supervision of GB. This article presents the results of this internship with a double objective. First, it shows how artificial life can be used to train young students with method and scientific rigor. Second, it presents Quentin's results, which are very real and worth sharing with the community. The article is structured chronologically, each section corresponding to a day of internship and to a stage of the research project. These five chronological sections are followed by two separate discussions. The first one deals with teaching the scientific process by means of artificial life; the second discusses the scientific results obtained on the causes of genome streamlining as it is observed in several species of bacteria. Finally, a Material and Methods section presents the tools used during the internship. All along this article we will make an extensive use of footnotes, to discuss technical points that either have not been taught to Quentin (because we considered they were too difficult) but that are important for the reader, or experimental results that have been recomputed after the internship to improve confidence ${ }^{1}$.

\section{Monday: Science always starts with a question}

The scientific method is known to start with a question, generally raised by a striking observation. Hence, experiencing the scientific method requires an observation, simple enough to be understandable by a naive person but also open enough to raise an interesting question. In the context of Quentin's internship, we chose to address the question of genome streamlining and to begin with the diver-

\footnotetext{
${ }^{1}$ All the simulations and statistical analyses were conducted anew by GB, MF, JRC and CK after the internship. In particular, we used a new Wild-Type - see methods - because the one used by Quentin was evolved in two steps $\left(10^{7}\right.$ generations in a population of 1024 individuals followed by $10^{6}$ generations in a population of 100 individuals), possibly biasing the results. We wanted to exclude this possibility before publication of the results.
} 
sity of genome sizes and structures in the bacterial kingdom.

Question: Difference in genome size across bacterial species. More than ten years ago, Giovannoni et al. (2005) published a graph comparing the sizes and structures of a large variety of bacterial genomes. Figure 1 shows a similar graph, as we explained it to Quentin.

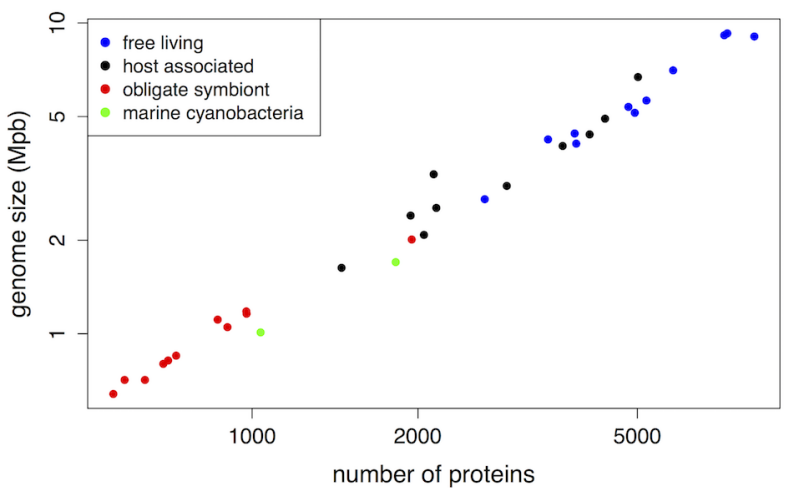

Figure 1: Genome size vs. number of protein coding genes for free living bacteria (blue), host-associated bacteria (black), obligate symbionts (red) and marine cyanobacteria living in very large populations (green). Data from NCBI.

The red dots on Figure 1 represent obligate symbionts. These bacteria have experienced a severe genome streamlining following their obligate association with a host (Wernegreen, 2002), raising the question of the causes of this streamlining. The genomes of free-living marine cyanobacteria show a similar pattern (Figure 1, green dots). Taking into consideration the profound difference in lifestyle, this similarity is quite striking.

A very popular theory to explain the large variety of genome sizes and structures has been proposed by Lynch and Conery (2003). It states that one of the main determinants of genome size is the effective population size $N e$. As it is well known in population genetics, $N e$ drives selection efficiency. Hence, in very large populations, genomes are under a strong selective pressure, preventing them from accumulating slightly deleterious sequences. On the opposite, small populations cannot avoid the proliferation of such elements, hence their large genome size. One of the strengths of this theory is its very elegant statement: by linking genome architecture to a single parameters $(\mathrm{Ne})$ it predicts a continuum of genome size and content very similar to what is observed on Figure 1. However, obligate symbionts do not fit easily with this theory. Indeed, because of their obligate status, they necessarily live in small subpopulations (each within a specific host individual). Starting from the observation that genome streamlining has occurred both in small and large population sizes (Batut et al., 2014), we proposed that Quentin could address the following question during his internship:

Starting from a wild-type genome, can different processes lead to genome streamlining and if so, is it possible to distinguish between them by observing the resulting genomes?

Hypothesis: Both large population size and high mutation rates can streamline genomes Once the question has been identified, science proceeds through experiments. However, experiments cannot be directly inferred from the question; we first have to propose hypotheses and then design the experiments to test these hypotheses. Many different hypotheses have been proposed to explain the striking genome streamlining in bacteria (reviewed in (Batut et al., 2014)). Here we will focus on two mechanisms that have been suggested to lead to genome streamlining: population size and mutation rate. Indeed, both have been independently suggested to impact genome size (Lynch and Conery, 2003; Lynch, 2006; Knibbe et al., 2007) but their respective effects have never been assessed experimentally. Moreover, both mechanisms have been proposed to impact the genetic structure differently: while population size has been proposed to act on non-coding sequences (because non-coding sequences are supposed to have slightly deleterious effects (Lynch and Conery, 2003)), mutation rates have been proposed to act on the whole genome, including coding and non-coding sequences (Knibbe et al., 2007). We thus proposed that Quentin test the following hypothesis:

Genome streamlining can be caused by changes in population sizes and/or by changes in mutation rates. These two mechanisms are likely to have different effects on coding and non-coding sequences.

Experimental design Being for experimental reasons or due to the limited time of the internship, it was not possible to perform in vivo experiments to test the aforementioned hypothesis. Now, provided that the experiments are well designed, it is possible to turn to artificial life and propose designs that enable (i.) to really perform scientific experiments (though in silico) (ii.) to get sound results after only a few hours of computation. We hence used the Aevol simulation platform (Knibbe et al., 2007; Batut et al., 2013; Liard et al., 2018) which has been specifically designed to study the evolution of genome architecture and complexity (see Methods).

Since artificial life enables to strictly follow the scientific method while minimizing the experimental and technical issues (e.g. here, how to modify the mutation rate of an organism?), we were able to teach Quentin the basis of the experimental method:

- Modify only one factor at a time,

- Make replicates to get statistical accuracy, 
- Compare the results with a control condition in which no factor has been changed,

- Record everything in your lab notebook ${ }^{2}$.

Importantly, we also discussed the issue of experimental costs which, while often neglected in teaching, strongly constrains the experiments in practice. Here, the experimental costs were exemplified by the available computational power Quentin had at his disposal during his internship. The experimental design phase was thus the occasion to present and discuss the actual scientific process and the importance of its different phases. In particular, we insisted on the fact that the experimental results must be gathered early enough such that enough time will remain to analyze them.

With all these elements in mind, Quentin designed two experiments, one to test the effect of mutation rate and one to test the effect of population size. Following the "in silico experimental evolution" strategy proposed by Batut et al. (2013), evolutionary runs started from a pre-evolved clone (the "Wild-Type"). Here, the same Wild-Type genome was used to seed all evolutionary runs. To test the effect of mutation rate, three series of evolutionary runs were performed: a series of Control runs were performed with the same parameters as those used to produce the Wild-Type, a series of $\mathrm{Mu}+$ runs were the mutation rate was increased, and a series of $\mathrm{Mu}$ - runs were the mutation rate was increased. For the population size experiment, the same control runs were used as for the mutation rate experiment, and a series of $\mathrm{N}+$ (resp. $\mathrm{N}^{-}$) runs were performed with increased (resp. decreased) population size. All simulations lasted 100,000 generations. Table 1 summarizes the five tested conditions. While designing the experiments, we discussed resource allocation. Here the problem was to estimate computation time to establish the number of repeats we were able to compute in a reasonable time. We initially chose to compute five repeats for each condition ${ }^{3}$. Finally, since in Aevol the computation time mainly depends on the population size, it was decided to allow more computational resources to the $\mathrm{N}+$ condition.

\section{Tuesday: Preliminary results}

The second day of the internship was almost entirely devoted to technical issues regarding Aevol output files, their location on the disk, how to collect them and the different tools available to analyze them; including the reconstruction of lineages (aevol_misc_lineage), the computation of lineages statistics (aevolmisc_ancestors_stats) and the visualization tools (aevol_misc_view). For plotting and data analysis, we decided to use gnuplot and LibreOffice/Calc as they are user-friendly.

\footnotetext{
${ }^{2}$ This was actually the first point we explained to Quentin at the beginning of the internship: we gave him a "notebook" and urged him to write down everything during his internship, including observations, hypotheses, experiments, results or simply ideas.

${ }^{3}$ All data presented in this paper have been computed with 10 repeats.
}

\begin{tabular}{|c||c|c|c|c|}
\hline $\begin{array}{c}\text { Exp. } \\
\text { name }\end{array}$ & $\begin{array}{c}\text { Mutation } \\
\text { rates }\end{array}$ & $\begin{array}{c}\text { Rearrangement } \\
\text { rates }\end{array}$ & $\begin{array}{c}\text { Pop. } \\
\text { size }\end{array}$ & $\begin{array}{c}\text { Nb } \\
\text { cores }\end{array}$ \\
\hline \hline Control & $1 \times 10^{-7}$ & $1 \times 10^{-6}$ & 100 & 1 \\
\hline $\mathrm{N}+$ & $1 \times 10^{-7}$ & $1 \times 10^{-6}$ & 400 & 4 \\
\hline $\mathrm{N}-$ & $1 \times 10^{-7}$ & $1 \times 10^{-6}$ & 25 & 1 \\
\hline $\mathrm{Mu}+$ & $4 \times 10^{-7}$ & $4 \times 10^{-6}$ & 100 & 1 \\
\hline $\mathrm{Mu}-$ & $2.5 \times 10^{-8}$ & $2.5 \times 10^{-7}$ & 100 & 1 \\
\hline
\end{tabular}

Table 1: Experimental design. Mutation and rearrangement rates are given in events.bp ${ }^{-1}$.generation ${ }^{-1}$. Column "Nb Cores" corresponds to the degree of parallelism used to compute each condition.

From the current state of the simulations, we were able to estimate the total computation time of the experiments and to reevaluate the number of repeats we could do during the internship. We hence decided to add two more repeats in order to increase the statistical accuracy of the results ${ }^{4}$

\section{Wednesday: Analyzing experimental results}

At the beginning of the third day of the internship, all computations were finished. We thus entered into a new phase of the scientific method: results analysis. Since Quentin was then autonomous enough with the experiments, we asked him to collect the characteristics of the best organism of each population at generation 100,000 for the five experimental conditions and to compute their mean values. Table 2 shows the corresponding results.

\begin{tabular}{|c||c|c|c|c|}
\hline $\begin{array}{c}\text { Exp } \\
\text { name }\end{array}$ & $\begin{array}{c}\text { Fitness } \\
\text { (mean) }\end{array}$ & $\begin{array}{c}\text { Genome } \\
\text { length } \\
\text { (mean) }\end{array}$ & $\begin{array}{c}\text { Coding } \\
\text { length } \\
\text { (mean) }\end{array}$ & $\begin{array}{c}\text { Non-coding } \\
\text { length } \\
\text { (mean) }\end{array}$ \\
\hline \hline Wild-Type & 0.00632 & $44,419 \mathrm{bp}$ & $12,235 \mathrm{bp}$ & $32,184 \mathrm{bp}$ \\
\hline \hline Control & 0.00643 & $44,044.4 \mathrm{bp}$ & $12,229.2 \mathrm{bp}$ & $31,815.2 \mathrm{bp}$ \\
\hline $\mathrm{N}+$ & 0.00766 & $37,116.9 \mathrm{bp}$ & $12,216.4 \mathrm{bp}$ & $24,900.5 \mathrm{bp}$ \\
\hline $\mathrm{N}-$ & 0.00292 & $46,067.6 \mathrm{bp}$ & $11,885.3 \mathrm{bp}$ & $34,182.3 \mathrm{bp}$ \\
\hline $\mathrm{Mu}+$ & 0.00468 & $33,693.8 \mathrm{bp}$ & $12,003.8 \mathrm{bp}$ & $21,690.0 \mathrm{bp}$ \\
\hline $\mathrm{Mu}-$ & 0.00641 & $45,226.7 \mathrm{bp}$ & $12,166.4 \mathrm{bp}$ & $33,060.3 \mathrm{bp}$ \\
\hline
\end{tabular}

Table 2: Mean characteristics of the best individuals in the populations at generation 100,000 for the five experimental conditions. These values are to be compared with those of the Wild-Type at generation 0 (first row).

Having computed the mean values for these characteristics, we entered a decisive step by asking Quentin the following question: Can the mean values, as shown in Table 2, be used to draw conclusions about the respective effects of population sizes and mutation rates? In Table 2, all mean values are different - actually means are always different but part of the difference is due to randomness and sampling

\footnotetext{
${ }^{4}$ This decision was not based on the p-values obtained so far since we did not compute them at this stage. Had we decided to add more runs until the p-values became significant, it would have been a form of p-hacking (Head et al., 2015). Note that the experiments presented here are replications of Quentin's ones and have been performed with a predefined experimental design (with ten repeats per condition).
} 
fluctuations. To clarify this point, we plotted the evolution of genome size along the line of descent of the best final organism for the 100,000 generations of the experiment. We used this temporal data to explain to Quentin that, especially when dealing with stochastic processes, mean values must be used with care as they don't account for an important element: dispersion. Now, when looking at these graphs, one could have the impression that $i$ ) an increased population size leads to genome streamlining (Figure 2) while a reduced population size tends to cause a slight increase in genome size (Figure 3), and that ii) an increased mutation rate leads to genome streamlining while a reduced mutation rate has no effect (figures not shown). Now the decisive question is "is this true?", opening a discussion about what does being true mean in experimental sciences?

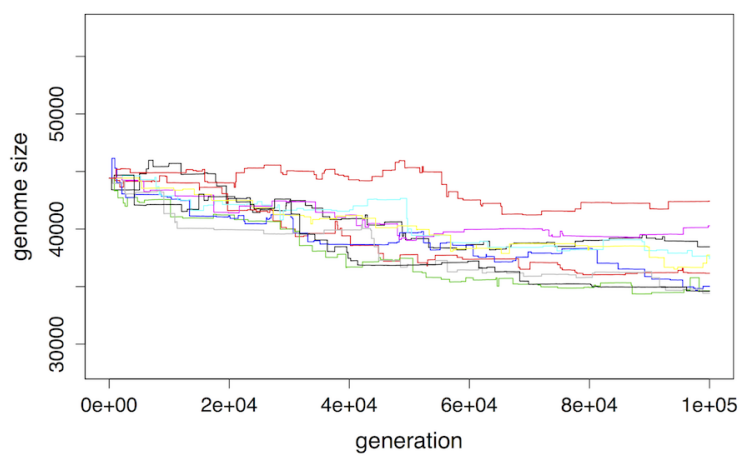

Figure 2: Variation of genome size in the lineage of the clones evolving within an increased population size $(\mathrm{N}+$ clones). Colors indicates the repeats.

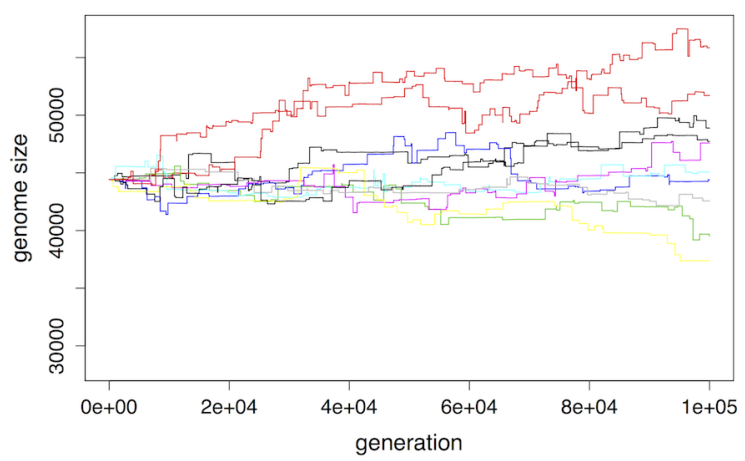

Figure 3: Variation of genome size in the lineage of the clones facing a reduced population size ( $\mathrm{N}-$ clones).

\section{Thursday: Statistical analysis}

Having conducted our experiments, it remained to be tested whether the data statistically supported our initial hypotheses, namely that both an increase in population size and an increase in mutation rates are likely to cause a genome reduction.
Given that we had sampled a few evolutionary runs among the infinite number of possible runs, we had only estimates of the mean evolved genome size in each condition. We explained to Quentin that if we were to replicate the experiment, we would sample different runs and hence obtain different mean estimates for each condition. Perhaps this time the observed mean genome size in the $\mathrm{N}+$ condition would not be smaller than the one in the Control condition. In other words, perhaps the smaller genomes we obtained in the $\mathrm{N}+$ condition was only due to sampling chance! But we observed a change of mean of more than $15 \%$, is sampling chance alone able to produce that? Actually, yes it is, and not necessarily with a low probability. Thus, we need to quantify the change in mean estimate that is expected by sampling chance only. Regarding the question of scientific truth, there is no such thing as experimental truth, only chances of being wrong when drawing conclusions from an experiment...

Given Quentin's age, it was not possible to enter a detailed discussion about random variables, normal distributions, statistical inference, parametric or non-parametric tests, etc. We instead decided to go for a semi-statistical, semi-graphical approach, in three steps:

Step 1: the Central-Limit Theorem We first explained to Quentin the basis of the Central Limit Theorem $\left(\mathrm{CLT}^{5}\right)$. The CLT tells us that if we replicate many times the procedure of sampling $n$ runs and computing the observed average genome size across the $n$ runs of the sample, and if we draw the histogram of the observed sample means, then we will get a bell shape ${ }^{6}$. The width of the bell tells us how much the sample mean is likely to change by sampling chance alone, from sample to sample.

Step 2: Confidence Intervals Statistical theory gives us a formula to estimate the width of the bell and to compute a so-called Confidence Interval (CI) for the mean. This formula depends both on the observed sample dispersion and on the sample size. In our case, with $n=10$ (i.e. 9 degrees of freedom), the $95 \%$ confidence interval is $\mathrm{CI} 95 \%=\left[\bar{x} \pm 2.262 \sqrt{\left(s^{2} / n\right)}\right]$, with $s^{2}=$ $\frac{1}{n-1} \sum\left(x_{i}-\bar{x}\right)$ and 2.262 coming from Student's t table for 9 degrees of freedom. A 95\% CI captures the true mean for $95 \%$ of the samples. We helped Quentin build a spreadsheet to compute $s^{2}$ and the CI95\% for each of the conditions.

\footnotetext{
${ }^{5}$ It would have been highly valuable to test it experimentally by e.g. computing more repeats in the Control condition. However, this was impossible in the limited duration of the internship.

${ }^{6}$ Actually, the random variable $\frac{\bar{X}-\mu}{\sigma / \sqrt{n}}$ has a standard normal distribution (i.e. normal with expected value 0 and variance 1 ), and the random variable $\frac{\bar{X}-\mu}{s / \sqrt{n}}$, where $s$ is the Bessel-corrected sample variance, has a Student's t-distribution with $n-1$ degrees of freedom.
} 
Step 3: Do confidence intervals overlap? From confidence intervals, we asked Quentin to find a way to identify interesting effects, i.e. those that are most likely not due to sampling chance alone. He decided to check whether confidence intervals overlap or not ${ }^{7}$. Based on this criterion, he concluded that "significant" effects were the following:

1. The genome of $\mathrm{Mu}+$ and $\mathrm{N}+$ are smaller than the genome of the Control (Figure 4).

2. The genomes of the $\mathrm{Mu}+$ and $\mathrm{N}$ - contain less coding sequences than the genome of the Control and the genome of the $\mathrm{N}+$ (Figure 5, top panel).

3. The non-coding length of $\mathrm{Mu}+$ and $\mathrm{N}+$ are lower than the non-coding length of the Control (Figure 5, bottom panel).

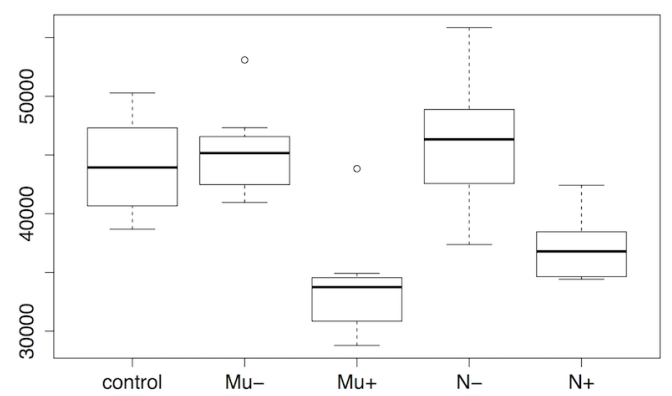

Figure 4: Genome size at generation 100,000 for the 5 conditions.

\section{Friday: Put the results into perspective}

One might think that the previous results, obtained after four days, close the scientific process. We chose to show Quentin that this is not the case, on the contrary! Friday was entirely devoted to discussion between Quentin, his supervisor and the rest of the team. He also presented his results to members of the team who had not followed his work. Our goal was to show Quentin that a scientific result must be put in perspective and confronted with the current state of knowledge. We also wanted to show him that communicating results and conclusions is an important part of a scientific work: a scientist must be able to present his results to the community, discuss them and possibly argue against opponents.

Last but not least for a youngster attracted by a scientific career, we discussed the qualities that are necessary to become a researcher, from the obvious (curiosity, rigor, intellectual honesty...) to qualities less often put forward but

\footnotetext{
${ }^{7}$ Quentin came up with a criterion that is actually used quite often by e.g. biologists, although this approach is not equivalent to performing a statistical test (Krzywinski and Altman, 2013). Here, we performed Kruskall-Wallis tests followed by post-hoc Dunnett tests to compare each condition to the control. We then applied a Bonferroni correction. The $\mathrm{p}$-values of the tests are presented in appendix.
}
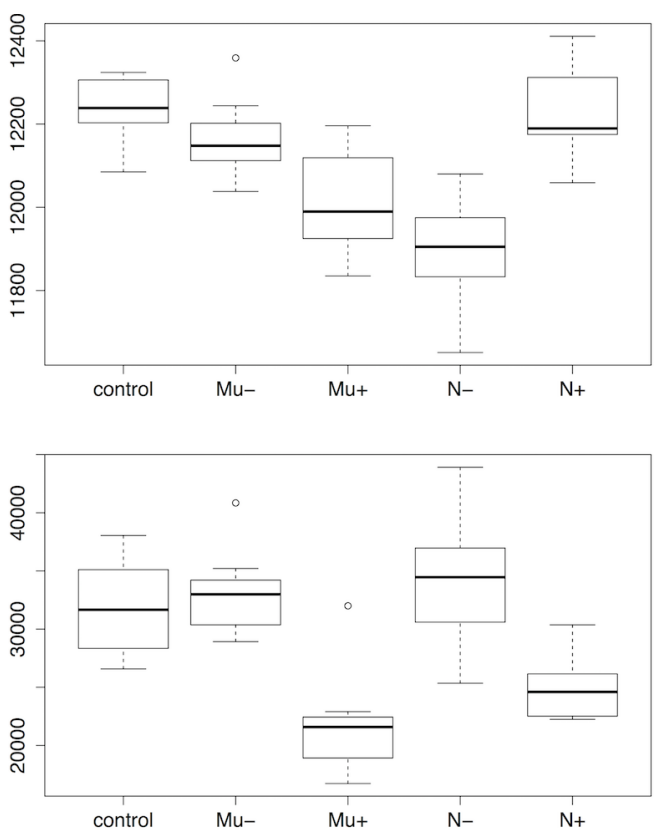

Figure 5: Size of the two main genomic compartments at generation 100,000 for the 5 conditions. Top: coding compartment. Bottom: non-coding compartment.

just as important: passion, pertinacity, scientific (and nonscientific) culture, or - fundamental for a young French boy - the level of English!

\section{Discussion}

During this internship - and in this article - we showed two things. First, that ALife could serve as a powerful pedagogical tool to teach the scientific method, including to young and untrained students. Second, that life-traits can strongly influence the length and structure of genomes. Below we separately discuss these two points.

\section{Using ALife to teach the scientific method}

Quentin's internship in the Beagle team illustrated the strength of ALife as a teaching tool. However, contrary to what is generally proposed, here ALife has not been used to teach biology or evolution but rather to teach the scientific method, its main tools and its main issues. We argue that this pedagogical usage of ALife is actually more straightforward than the former usage. Indeed, using simulation to teach biology requires that the student have preliminary understanding of difficult, abstract and actually fuzzy concepts linked to modelling of biological systems (models being, by essence, different from the system they model). This is even more so for artificial life that intends to model life "as it could be" (i.e. in its whole generality) rather than as it is. It also implicitly implies to change the destination of the models (from mere research to teaching) and its user. Now, 
following e.g. Minsky (1965) definition of a model ("To an observer $B$, an object $A *$ is a model of an object $A$ to the extent that $B$ can use $A^{*}$ to answer questions that interest him about A"), it is clear that both the observer $B$ and the destination (i.e. the "questions that interest him") of a model are central in the complex relationship that links the model object $A^{*}$ to the original object $A$. In a word, changing the destination and the user of the model results in such a deep alteration of the $A / A^{*}$ relationship that it generally implies changing... the model! This is indeed the process the Avida team engaged through the development of Avida-ED (Speth et al., 2009).

These difficulties vanish when ALife is used to teach the basis of the scientific method, be it during an internship or a labwork. Indeed, in this case, the student is engaged in a scientific process: he/she must answer a question about the model behaviour, exactly as the original user of the model would. Hence there is no change in the model destination and the user change is only minor since the student actually plays the role of a scientist. As a matter of fact, during Quentin's internship, we did not encounter any conceptual issues regarding the differences between the model and the "real" system. This is simply due to the studied object being Aevol and not what it models. The relationship between the model and the real world was indeed discussed during the internship but that was at the very end (on Friday) and it did not need to be accepted a priori. When Quentin was asked to summarize what he had learned during his internship, his answer was: "During my five day work experience with Inria, I have been able to observe and to learn the reality of research and what are the different tasks of this job: to put forward hypotheses, to experiment, to analyze and to publish results. I have learned what are the main qualities of a researcher and what are the studies leading to this job. In only five days, thanks to simulation, I obtained results, I was able to analyze them and then to present them. I noticed that, in teamwork, its very important to have good relations between team members". Of course, he has also learned a lot about evolution, genomics and genetics. But this appears to be less important that the insights into the scientific process itself...

Of course, we - Quentin and us - encountered difficulties during the internship. But most of them were technical, not directly related to the use of Artificial Life. Importantly, none of them proved to be crucial and none compromised the learning process. In fact, the main difficulty encountered was the visualization of the raw data and the visualization of the results of the statistical analyzes. Indeed, all the figures presented in this article were made using $\mathrm{R}$ but this software was clearly unusable in the context of such a short educational process. Even though we were able to work around the problem, it would clearly have been desirable to have a simple tool allowing Quentin - a naive user on that matter to manipulate and visualize the data autonomously.
When teaching the scientific method, ALife proved to have valuable advantages. Here, we will focus on the two main ones. First ALife relies on in silico experiments, which is twice an advantage as it allows for fast experiments that can also be easily replicated (the replication effort being supported by the computer rather than by the student). In the case of Quentin's internship, a single computer - though a relatively powerful one - was used, enabling him to conduct 5 series of 7 runs, in little more than $24 \mathrm{~h}$. Second, using ALife, one can propose internships or labworks focusing on open scientific questions, for which neither the students nor the mentors have a definitive answer. This creates a strong initial motivation and allows to maintain it all along the process as both the students and the mentors are likely to be surprised - possibly negatively - by the results. In the case of Quentin's internship, the results shed an interesting light on the process of genome streamlining that deserves to be discussed on its own.

\section{How to reduce a genome?}

Genome reduction is common in Nature but its causes are still elusive as biological data suggest that genome reduction could be either neutral or adaptive (Wolf and Koonin, 2013). Our results suggest that at least two distinct mechanisms can lead to genome reduction and that they are distinguishable by their effect on coding sequences. We also show that these mechanisms are triggered by two different causes: increased mutation rates or increased population size.

Interestingly, the effects we observed here fit remarkably well with what is observed in streamlined bacteria. Indeed, obligate symbionts have an elevated mutation rate (Itoh et al., 2002) while marine cyanobacteria live in very large populations (Batut et al., 2014). Both have undergone genome streamlining but the reduction is more pronounced in obligate symbionts (Figure 1), as in our simulations. Moreover, in these two families, the reduction seemed to have impacted differently the different genomic compartments. Marine cyanobacteria have mainly lost noncoding or duplicated elements, as examplified by Pelagibacter ubique, one of the smallest genome of free living bacteria. Its genome is characterized by a very small fraction of non-coding DNA (less that 5\%) and the quasi-absence of redundancy in coding sequences while all metabolic pathways are still present (Giovannoni et al., 2005). By contrast, the genome of Buchnera aphidicola, an aphid endosymbiont has lost $90 \%$ of its genome (compared to E. coli, one of its close relatives), lost several metabolic pathways but, strikingly, has conserved $15 \%$ of non-coding sequences, a proportion similar to what is observed in E. coli (Batut et al., 2013).

The results presented here don't allow to identify the causal link between mutation rates, population size and genomes size. However, taking advantage of the model characteristics - and of our previous results with Aevol, we can 
exclude some mechanisms and put others forward. Typically, many authors suggest that genome size may be governed by mutational biases, selection for optimized physiological traits (cell size, replication time, energetic costs...) or by transposable elements activities. These are all excluded by our simulation parameters or by Aevol itself. Moreover, in Aevol, there is no cost associated to non-coding sequences. Hence, the influence of population size on genome length cannot be linked to the deleterious effect of noncoding sequences as argued by (Lynch and Conery, 2003).

In the absence of direct selective effects or mutational biases, we hypothesize that genome size is driven by indirect selective constraints, namely by selection for robustness (Wilke et al., 2001). Indeed, as already shown by Knibbe et al. (2007), selection for robustness links genome size to mutation rates, the higher the latter, the smaller the former. We hypothesize that the same phenomenon also explains the influence of population size: in large populations, the selection strength is higher, increasing the pressure for robustness, thereby favouring smaller genomes. The striking question is then to explain why both phenomena act similarly on non-coding sequences but differently on coding sequences (for which only an elevated mutation rate induces a reduction). We propose that, exactly like selection for fitness, selection for robustness may act positively (selecting more robust clones) or negatively (eliminating clones that are not robust enough - aka purifying selection). Now, in the case of an increased mutation rate, the error threshold (Eigen and Schuster, 1977) moves down and some individuals may find themselves over this crucial threshold. In this case, selection will purify the population from these individuals, retaining only those that reduced their genome, whatever the elements they have lost (including coding and non-coding sequences). On the opposite, in case of an elevated population size, the error threshold does not move down and individuals are still robust enough to maintain their fitness. However, providing adaptive mutations are rare (which is the case here), this results in a positive selection for robustness. In this case, individuals must retain their fitness (hence their coding sequences) and the only way to increase their robustness is to get rid of non-coding elements. To the best of our knowledge, these two contrasting effects of selection for robustness had never been identified before. Not content with illustrating the interest of using artificial life to teach the scientific method, our results also show that interesting scientific insights can be gathered meanwhile and open the exciting perspectives of characterizing these two effects in our experiments (by e.g. measuring robustness levels along the evolutionary path), in different conditions and in different systems.

\section{Material and methods Simulation platform}

All simulations were run using the regular Aevol model, version 5.0, as available on the platform website (www. aevol.fr). Since Aevol has been extensively described elsewhere (Knibbe et al., 2007; Batut et al., 2013) we will not detail it here and focus only on its core principles and on the elements that are specifically of interest for this paper. Figure 6 shows the main components of the model. Aevol simulates a population engaged in a generational process (Fig. 6.A). Each individual is described by a circular doublestrand genomic sequence whose structure closely models a bacterial genome (including non-coding sequences, transcription and translation initiation sequences, open-reading frames...) making Aevol an ideally suited platform to study the evolution of genome length and structure. This genome is decoded into a $[0: 1] \rightarrow[0: 1]$ mathematical function which proximity with a target function gives the fitness of the individual (Fig. 6.B). Individuals replicate locally (Fig. 6.C) and, importantly for the present study, Aevol implements a large variety of variation operators (Fig. 6.D) including mutations (base switches, small insertions and small deletions) and chromosomal rearrangements (duplications, deletions, translocations and inversions). All variation operators can be tuned independently, making the platform an ideally suited tool to study indirect selection for mutational robustness.

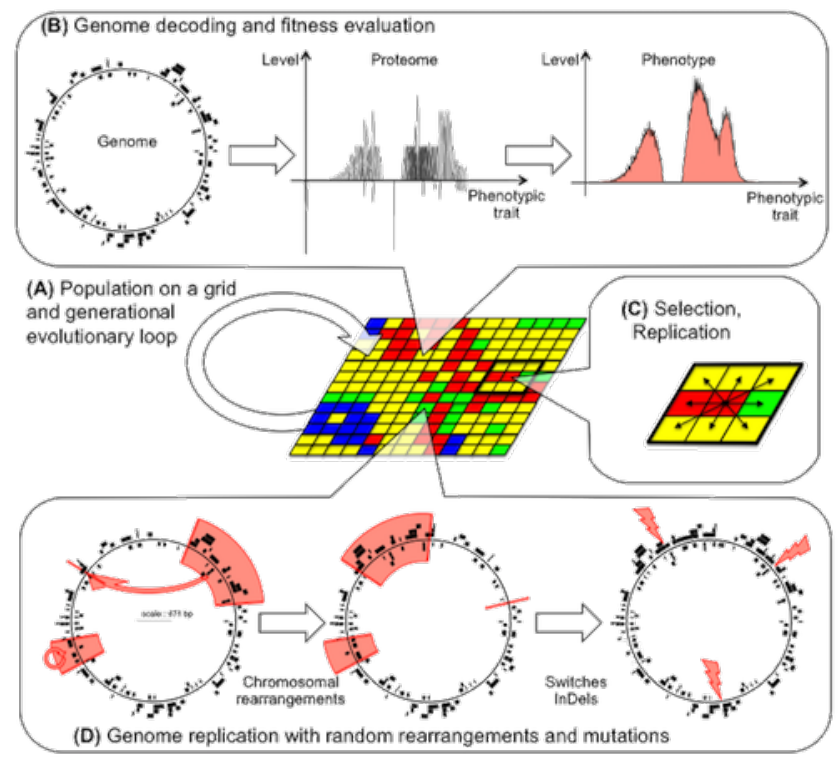

Figure 6: The Aevol model (figure from (Liard et al., 2018)). (A) Population on a grid and evolutionary loop. (B) Overview of the genotype-to-phenotype map. (C) Local selection process with a Moore neighborhood. (D) Variation operators include chromosomal rearrangements and local mutations. 


\section{Evolution of the Wild-Type strain}

To study genome streamlining, we had to give Quentin an initial organism (the "Wild-Type") that was evolved prior to the internship. In the experiments presented here the wild-type evolved with a population of 100 individuals, a mutation rate of $10^{-7}$ events.bp ${ }^{-1}$.generation ${ }^{-1}$ for each of the three kinds of mutations and a rearrangement rate of $10^{-6}$ events.bp $^{-1}$.generation ${ }^{-1}$ for each of the four kinds of rearrangements. We used an unusually small population size in order to allow for fast experiments.

In order to ensure that the genome size and structure of the wild-type have reached a steady state, we let it evolve for 10 million $\left(10^{7}\right)$ generations in constant conditions as preliminary results have shown that the genome size did not stabilize before $5.10^{6}$ generations (data not shown). Note that the evolution of genome size and genome structure in the Control conditions (see Table 2) confirmed that the genome was stabilized. We then extracted the genome of the best individual at the last generation. It contains 44,419 bp, 32,184 of which are non-coding and 12,235 coding. It encodes 146 genes transcribed on 108 coding mRNA, approximately half of which being polycistronic. Its fitness is 0.00632 . Note that the proportion of non-coding sequences is rather high in this organism, probably because of the small population size.

\section{Experimental design}

Starting from the wild-type genome, we used the aevol_create tool to initialize a clonal population of wild-types with specific parameters (population size, mutation and rearrangement rates, see Table 1). This procedure allowed us to avoid sampling issues when changing the population size.

All simulations were then performed on an Intel Xeon CPU with 32-cores at $2 \mathrm{Ghz}$ with 32 Go RAM that Quentin had at his entire disposal for the whole duration of the internship. With this configuration, all the computations required approximately $48 \mathrm{~h}$.

\section{Appendix: results of the statistical analyses}

Results of the Kruskall-Wallis (KW) and post-hoc Dunnett tests for effects of mutation rates and population size on genome size, coding length and non-coding length. Dunnett $\mathrm{p}$-values under 0.017 are in bold face (accounting for $\mathrm{p}<0.05$ with a Bonferroni correction for three responses).

\section{Genome size:}

KW on mutation rate: $\chi^{2}=16.694, \mathrm{df}=2$, p-value $=0.0002371$ Dunnett: $\mathrm{Mu}$ - vs. Control: 0.7205; Mu+ vs. Control: 4.4e-06 KW on Population size: $\chi^{2}=15.36, \mathrm{df}=2$, p-value $=0.000462$ Dunnett: N-vs. Control: 0.4661; N+ vs. Control: $\mathbf{0 . 0 0 2 0}$

\section{Coding length:}

KW on mutation rate: $\chi^{2}=13.388, \mathrm{df}=2$, p-value $=0.001238$ Dunnet: $\mathrm{Mu}-$ vs. Control: 0.2833; Mu+ vs. Control: 4.9e-05 KW on population size: $\chi^{2}=19.311, \mathrm{df}=2, \mathrm{p}$-value $=6.407 \mathrm{e}-05$ Dunnett: N- vs. Control: 3.5e-07; N+ vs. Control: 0.9523
Non-coding length:

KW on mutation rate: $\chi^{2}=16.498, \mathrm{df}=2, \mathrm{p}$-value $=0.0002615$

Dunnett: $\mathrm{Mu}-$ vs. Control: 0.6955; Mu+ vs. Control: 6e-06

KW on population size: $\chi^{2}=15.801, \mathrm{df}=2$, p-value $=0.0003705$

Dunnett: N-vs. Control: 0.3650; N+ vs. Control: $\mathbf{0 . 0 0 2 2}$

\section{References}

Batut, B., Knibbe, C., Marais, G., and Daubin, V. (2014). Reductive genome evolution at both ends of the bacterial population size spectrum. Nature Reviews Microbiology, 12(12):841.

Batut, B., Parsons, D. P., Fischer, S., Beslon, G., and Knibbe, C. (2013). In silico experimental evolution: a tool to test evolutionary scenarios. BMC bioinformatics, 14(15):S11.

Eigen, M. and Schuster, P. (1977). A principle of natural selforganization. Naturwissenschaften, 64(11):541-565.

Giovannoni, S. J., Tripp, H. J., Givan, S., Podar, M., Vergin, K. L., Baptista, D., Bibbs, L., Eads, J., Richardson, T. H., Noordewier, M., et al. (2005). Genome streamlining in a cosmopolitan oceanic bacterium. science, 309(5738):12421245 .

Head, M. L., Holman, L., Lanfear, R., Kahn, A. T., and Jennions, M. D. (2015). The extent and consequences of p-hacking in science. PLOS Biology, 13(3):1-15.

Itoh, T., Martin, W., and Nei, M. (2002). Acceleration of genomic evolution caused by enhanced mutation rate in endocellular symbionts. Proceedings of the National Academy of Sciences, 99(20):12944-12948.

Knibbe, C., Coulon, A., Mazet, O., Fayard, J.-M., and Beslon, G. (2007). A long-term evolutionary pressure on the amount of noncoding dna. Mol. Biol. Evol., 24(10):2344-2353.

Krzywinski, M. and Altman, N. (2013). Points of significance: error bars. Nature methods, 10(10):921.

Liard, V., Parsons, D., Rouzaud-Cornabas, J., and Beslon, G. (2018). The complexity ratchet: Stronger than selection, weaker than robustness. In Artificial Life Conference Proceedings, pages 250-257. MIT Press.

Lynch, M. (2006). Streamlining and simplification of microbial genome architecture. Annu. Rev. Microbiol., 60:327-349.

Lynch, M. and Conery, J. S. (2003). The origins of genome complexity. Science, 302(5649):1401-1404.

Minsky, M. (1965). Matter, mind and models. In Proceedings of IFIP Congress, Spartan Books, Wash. DC, pages 45-49.

Speth, E. B., Long, T. M., Pennock, R. T., and Ebert-May, D. (2009). Using avida-ed for teaching and learning about evolution in undergraduate introductory biology courses. Evolution: Education and Outreach, 2(3):415.

Wernegreen, J. J. (2002). Genome evolution in bacterial endosymbionts of insects. Nature Reviews Genetics, 3(11):850.

Wilke, C. O., Wang, J. L., Ofria, C., Lenski, R. E., and Adami, C. (2001). Evolution of digital organisms at high mutation rates leads to survival of the flattest. Nature, 412(6844):331.

Wolf, Y. I. and Koonin, E. V. (2013). Genome reduction as the dominant mode of evolution. Bioessays, 35(9):829-837. 